\title{
Spin models of quasi-1D quantum ferrimagnets with competing interactions
}

\author{
N.B. Ivanov ${ }^{1,2}$ \\ 1 Institute of Solid State Physics, Bulgarian Academy of Sciences, \\ 72 Tzarigradsko chaussee, 1784 Sofia, Bulgaria \\ 2 Fakultät für Physik, Universität Bielefeld, D-33501 Bielefeld, Germany
}

Received June 19, 2009

\begin{abstract}
We present a brief survey of the recent theoretical work related to generic Heisenberg spin models describing quasi-one-dimensional quantum ferrimagnets. The emphasis is on quantum chains and ladders with strong competing interactions, such as the frustrated $J_{1}-J_{2}$ chain with alternating $(1,1 / 2)$ spins, the spin-1/2 diamond chain with four-spin cyclic couplings, and some generic types of mixed-spin ladders with geometric frustration. As a rule, the discussed models exhibit rich quantum phase diagrams and provide some interesting examples of one-dimensional magnetic-paramagnetic quantum phase transitions. A number of open problems in the reviewed research area are discussed.
\end{abstract}

Key words: quantum spin chains, ferrimagnets, frustration

PACS: $75.10 . J m, 75.10 P q, 75.45 .+j$

\section{Introduction}

Ferrimagnets are unsaturated magnetic materials exhibiting a net ferromagnetic (FM) moment, as a rule resulting from magnetic sublattices with different magnetic ions and/or different number of magnetic sites [1]. During the past two decades it has become possible to synthesize a large variety of quasi-one-dimensional (1D) mixed-spin compounds with ferrimagnetic properties [2]. Most of these materials are bimetallic molecular magnets containing two different transition metal ions per unit cell, which are alternatively distributed on the lattice (see figure 1). $\mathrm{MnCu}$ $(\text { dto })_{2}\left(\mathrm{H}_{2} \mathrm{O}\right)_{3} \cdot 4.5 \mathrm{H}_{2} \mathrm{O}$ (dto = dithiooxalato) is the first structurally characterized ferrimagnetic chain $[3,4]$. Two families of ferrimagnetic chains are described by $\mathrm{ACu}(\mathrm{pba})\left(\mathrm{H}_{2} \mathrm{O}\right)_{3} \cdot \mathrm{nH}_{2} \mathrm{O}(\mathrm{pba}=$ 1,3-propylenebis) and $\mathrm{ACu}(\mathrm{pbaOH})\left(\mathrm{H}_{2} \mathrm{O}\right)_{3} \cdot \mathrm{nH}_{2} \mathrm{O}$ (pbaOH $=$ 2-hydroxo-1,3 - propylenebis), where $\mathrm{A}=\mathrm{Ni}, \mathrm{Fe}, \mathrm{Co}$, and $\mathrm{Mn}[5-7]$. The cited pioneer studies have stimulated further extensive experimental research on heterometallic and homometallic ferrimagnetic chains [8-13]. Another class of ferrimagnetic materials are the so-called topological ferrimagnets. The homometallic magnetic compound $\mathrm{A}_{3} \mathrm{Cu}_{3}\left(\mathrm{PO}_{4}\right)_{4}(\mathrm{~A}=\mathrm{Ca}, \mathrm{Sr}, \mathrm{Pb})[11]$ is a quasi-1D example of such materials. In this compound, the $\mathrm{Cu}^{2+}$ ions form diamond chains with strongly coupled trimers bridged by oxygen ions. Similar magnetic structures appear in the magnetic compound $\mathrm{Cu}_{3}\left(\mathrm{CO}_{3}\right)_{2}(\mathrm{OH})_{2}[12,13]$ known as azurite. In addition, there are a number of experimental works on quasi-1D organic and inorganic magnetic materials with similar structures and ferrimagnetic properties [14]. Apart from pure scientific interest, the discussed systems possess a potential for various technological applications.

The discussed experimental research has established the basis for future theoretical studies on 1D quantum spin models exhibiting quantum ferrimagnetic states. Nowadays, this is one of the areas in the framework of the intensive research on 1D magnetism [15]. Below we present a brief survey of the theoretical results in this hot area, the emphasis being on some basic quantum spin models of quasi-1D quantum ferrimagnets with competing interactions. A generic spin model of the 1D quantum ferrimagnet is the Heisenberg spin chain with AFM nearest-neighbor exchange interactions and two different alternating spins $S_{1}$ and $S_{2}\left(S_{1}>S_{2}\right)$. The extreme quantum variant 


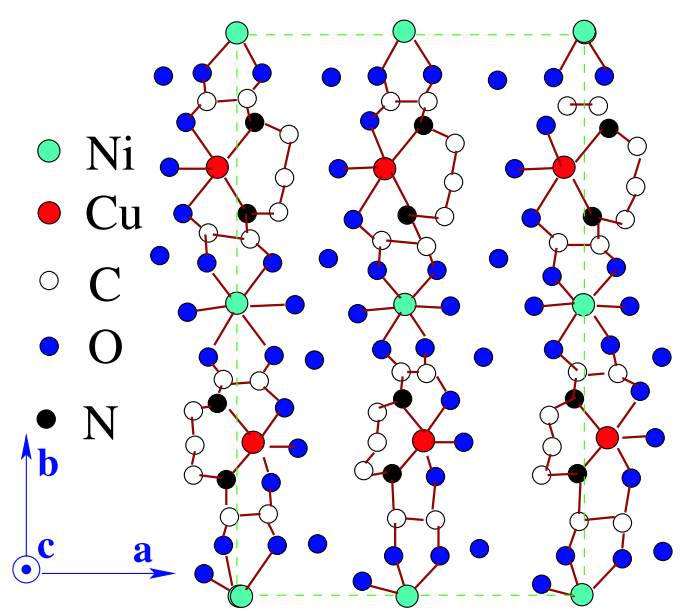

(a)

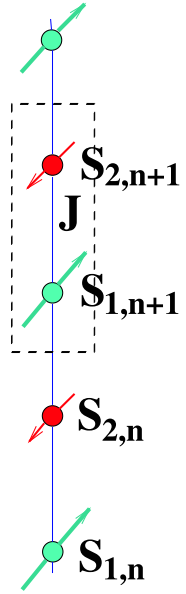

(b)

Figure 1. (a) Structure of the quasi-one-dimensional bimetallic compound $\mathrm{NiCu}(\mathrm{pba})\left(\mathrm{H}_{2} \mathrm{O}\right)_{3} \cdot 2 \mathrm{H}_{2} \mathrm{O}$ with alternating site spins $S_{1}=S_{\mathrm{Ni}}=1$ and $S_{2}=S_{\mathrm{Cu}}=\frac{1}{2}$ along the axis b. Hydrogen atoms are omitted for clarity [9]. (b) The classical Néel state of the mixed-spin AFM chain model (1) describing the bimetallic compound $\mathrm{NiCu}(\mathrm{pba})\left(\mathrm{H}_{2} \mathrm{O}\right)_{3} \cdot 2 \mathrm{H}_{2} \mathrm{O}$.

of this model $\left(S_{1}=1, S_{2}=1 / 2\right)$ is used in section 2 to survey some basic ground-state and thermodynamic properties of $1 \mathrm{D}$ quantum ferrimagnets. The emphasis in section 3 is on the quantum phase diagrams of a few generic Heisenberg spin models describing 1D quantum ferrimagnets with strong competing interactions, such as the frustrated $J_{1}-J_{2}$ chain with alternating $(1,1 / 2)$ spins, the spin-1/2 diamond chain with four-spin cyclic couplings, and some basic types of mixed-spin ladders with geometric frustration. The last section contains some conclusions.

\section{The antiferromagnetic mixed-spin Heisenberg chain}

A generic spin model of the 1D quantum ferrimagnet is the Heisenberg spin chain with AFM nearest-neighbor exchange interaction $(J>0)$ and alternating spins $S_{1}$ and $S_{2}\left(S_{1}>S_{2}\right)$, described by the Hamiltonian

$$
\mathcal{H}=J \sum_{n=1}^{L}\left(\mathbf{S}_{1, n}+\mathbf{S}_{1, n+1}\right) \cdot \mathbf{S}_{2, n}-\mu_{\mathrm{B}} H \sum_{n=1}^{L}\left(g_{1} S_{1, n}^{z}+g_{2} S_{2, n}^{z}\right) .
$$

The integers $n$ number the unit cells, each containing two lattice spacings and two kinds of quantum spin operators $\mathbf{S}_{1, n}$ and $\mathbf{S}_{2, n}$ characterized by the quantum spin numbers $S_{1}$ and $S_{2}\left(S_{1}>S_{2}\right)$, respectively. $g_{1}$ and $g_{2}$ are the $g$-factors of the site magnetic moments, $\mu_{\mathrm{B}}$ is the Bohr magneton, and $H$ is an external uniform magnetic field applied along the $z$ direction. As an example, the following parameters of the Hamiltonian (1) have been extracted from magnetic measurements on the recently synthesized quasi-1D bimetallic compound $\mathrm{NiCu}(\mathrm{pba})\left(\mathrm{D}_{2} \mathrm{O}\right)_{3} \cdot 2 \mathrm{D}_{2} \mathrm{O}$ [9] with a structure which is similar to the one shown in figure 1: $\left(S_{1}, S_{2}\right) \equiv\left(S_{\mathrm{Ni}}, S_{\mathrm{Cu}}\right)=(1,1 / 2), J / k_{\mathrm{B}}=$ $121 K, g_{1} \equiv g_{\mathrm{Ni}}=2.22, g_{2} \equiv g_{\mathrm{Cu}}=2.09$. Henceforth we suggest $g_{1}=g_{2} \equiv g$ and use the Planck constant $\hbar$, the Boltzmann constant $k_{\mathrm{B}}$, and the lattice spacing $a_{0}$ as unites.

\subsection{Ground-state properties and excitations}

\subsection{1. $H=0$}

Let us start with the case $H=0$. According to Lieb-Mattis' theorem [16], for $H=0$ the ground state of the bipartite model (1) has a total spin $S_{\mathrm{T}}=\left(S_{1}-S_{2}\right) L, L$ being the number of unit cells, so that it is necessarily long-range ordered. Such ferrimagnetic ground states may also be referred 
to as quantized ferrimagnetic states since the FM moment is quantized in integral (or half-integral) multiples of the number of unit cells $L$. As explicitly demonstrated below (section 3 ), strong enough competing interactions can suspend this quantization rule in some regions of the parameter space where the long-range ferrimagnetic order still survives. Since the model has a magnetically ordered ground state, this makes the 1D problem amenable to the spin-wave theory (SWT) approach [17].

Valuable qualitative information about the ground state and low-lying excitations can be extracted already from the linear spin-wave theory (LSWT) [18-21]. The on-site magnetizations $m_{1}=\left\langle S_{1, n}^{z}\right\rangle$ and $m_{2}=-\left\langle S_{2, n}^{z}\right\rangle\left(m_{1}-m_{2}=S_{1}-S_{2}\right)$ are parameters of the quantum ferrimagnetic phase keeping information about the long-range spin correlations. LSWT implies that in the extreme quantum case $\left(S_{1}, S_{2}\right)=(1,1 / 2)$ the quantum spin fluctuations reduce substantially the classical on-site magnetizations $\left(m_{2} / S_{2} \approx 0.39\right)$. Notice, however, that due to the broken sublattice symmetry, in the mixed-spin models there appear important first-order corrections to $m_{1}$ and $m_{2}$ resulting from two-boson interactions in the bosonic Hamiltonian [22]. Up to second order in $1 / S_{2}$ (in respect to the linear approximation), SWT series gives the precise result $m_{2}=0.29388$ [23], to be compared with the density-matrix renormalization-group (DMRG) estimate $m_{2}=0.29248$ [20]. Another interesting peculiarity of the mixed-spin chain is the extremely small correlation length (smaller than the unit cell) of the short-range spin fluctuations [19,20]. This observation explains the good quantitative description of the model achieved with the variational approach using matrix-product states [24,25].

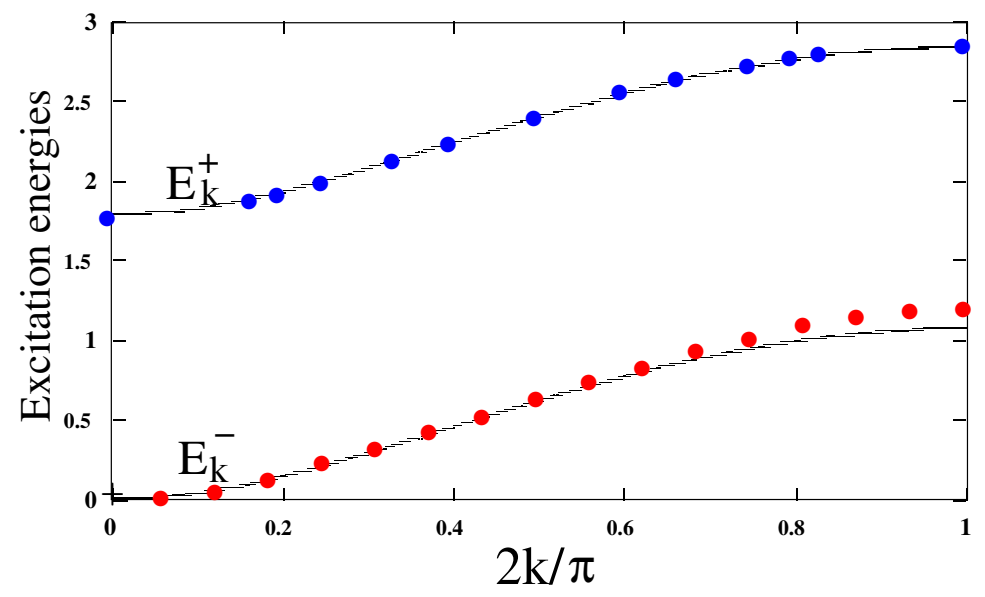

Figure 2. Dispersions of the one-magnon excitations $\left(E_{k}^{ \pm}\right)$in the system $\left(S_{1}, S_{2}\right)=(1,1 / 2)$ calculated up to second order in the $1 / S_{2}$ relative to the linear approximation [27]. The points in $E_{k}^{ \pm}$show numerical ED results for periodic chains (for $E_{k}^{+}$) and QMC results (for $E_{k}^{-}$) [21].

Turning to the excitation spectrum, SWT predicts two types of low-lying magnon excitations $E_{k}^{ \pm}$, respectively, in the subspaces with $z$ components of the total spin $S_{\mathrm{T}}^{z}=S_{\mathrm{T}} \pm 1$ (see figure 2). In the long wavelength limit $k \ll 1$, the FM mode $E_{k}^{-}$takes the Landau-Lifshitz form

$$
E_{k}^{-}=\frac{\varrho_{\mathrm{s}}}{M_{0}} k^{2}+\mathcal{O}\left(k^{4}\right)
$$

where $\varrho_{\mathrm{s}}=J S_{1} S_{2}$ is the spin stiffness constant [26] and $M_{0}=\left(S_{1}-S_{2}\right) / 2$ is the linear density of the net FM moment. This form of the Goldstone modes is typical of Heisenberg ferromagnets, and reflects the fact that the FM order parameter is itself a constant of the motion. As demonstrated below, the parameters $\varrho_{\mathrm{s}}$ and $M_{0}$ play a basic role in the low-temperature thermodynamics of the model. On the other hand, the AFM branch $E_{k}^{+}$is gapped, with a minimum at $k=0$ given by $\Delta^{+}=2\left(S_{1}-S_{1}\right) J$ in a LSWT approximation. Note, however, that the LSWT estimates for $\varrho_{\mathrm{s}}$ and the AFM gap $\Delta^{+}$are further renormalized by the boson-boson interactions [27]: In the extreme quantum system $\left(S_{1}, S_{2}\right)=(1,1 / 2)$, already the first order corrections in $1 / S_{2}$ give the results $\varrho_{\mathrm{s}} /\left(J s_{1} s_{2}\right)=0.761$ and $\Delta^{(+)}=1.676 J$. The quantum Monte-Carlo (QMC) result for this 
parameter 1.759 [21] clearly indicates the importance of the $1 / S_{2}$ corrections. Precise estimates for the parameters of the $(1,1 / 2)$ chain have also been obtained by using cluster series expansions [28]: $m_{2}=0.292487(6), \Delta^{+} / J=1.7591(6)$ and $\varrho_{\mathrm{s}} /\left(J s_{1} s_{2}\right)=0.831(5)$.

\subsection{2. $H \neq 0$}

The introduction of a Zeeman term in equation (1) leaves all eigenstates with a given $z$ component of the total spin $S_{\mathrm{T}}^{z}$ invariant, while shifting the eigenenergies by $h S_{\mathrm{T}}^{z}$, where $h=g \mu_{\mathrm{B}} H$. In particular, the one-magnon states $E_{k}^{ \pm}$, carrying magnetizations \pm 1 , change to $E_{k}^{ \pm}(h)=E_{k}^{ \pm} \mp h$, i.e, the Zeeman term introduces a gap $\Delta^{-}(h)=h$ for the FM branch and reduces the gap of the AFM branch, $\Delta^{+}(h)=\Delta^{+}-h$. If the magnetic field exceeds the critical value $h=h_{\mathrm{c} 1}=\Delta^{+}$, the AFM gap closes and the system enters a critical phase which is expected to be a kind of Luttinger liquid, in analogy with the behavior of other gapped spin models like the spin-1 chain and the spin-1/2 ladder [29-31]. The critical phase terminates at a second critical field $h=h_{\mathrm{c} 2}=3 J$ at which the system becomes fully polarized [32]. An accurate description of the mixed-spin $(1,1 / 2)$ Heisenberg chain $(H \neq 0)$ in the critical phase $h_{\mathrm{c} 1}<h<h_{\mathrm{c} 2}$ has been achieved by a mapping to an effective spin-1/2 XXZ chain in external magnetic field which uses variational matrix-product states [25]. Interestingly, such a critical phase seems to be characteristic of the entire class of mixed-spin $\left(S_{1}, S_{2}\right)$ anisotropic XXZ Heisenberg chains with easy-plane anisotropy and short-range interactions. An extensive numerical analysis, using exact-diagonalization (ED) results for $(1,1 / 2)$ and $(3 / 2,1 / 2)$ periodic chains, suggests universal critical properties for these chains in the entire interval from the FM to the ferrimagnetic isotropic points [33]. Along this phase, the critical fluctuations are ruled by a conformal field theory of Gaussian type with a topological charge $c=1$.

The discussed one-magnon excitations control the magnetization process of the mixed-spin chain. Since the lowest excitation increasing the FM moment $M=\left\langle S_{1, n}^{z}+S_{2, n}^{z}\right\rangle$ exhibits an energy gap, the magnetization curve $M(H)$ has a plateau at $M=S_{1}-S_{2}$. This plateau phase, however, appears in the related classical system as well. In this connection, it is interesting to examine the role of different anisotropies which may appear in real materials. As to the exchange anisotropy, it occurred that the quantum effects simply stabilize the plateau at $M=S_{1}-S_{2}$ against the XY anisotropy, i.e., the effect is reduced to quantitative changes of the classical result [34]. A clear indication for the quantum origin of the discussed magnetization plateau was provided for the $(1,1 / 2)$ chain with a single-ion anisotropy for spin-1 sites, $D\left(S_{1, n}^{z}\right)^{2}$, which is even more realistic for the existing mixed-spin materials [35]. It was revealed, in particular, that the mechanism of the $M=1 / 2$ plateau can change from a Haldane to a large- $D$ type through a Gaussian quantum critical point, estimated as $D / J=1.114$, which is a kind of justification for the quantum origin of the $M=1 / 2$ plateau in this system. Two plateau phases $(M=1 / 2)$ with different parities have also been studied in a frustrated mixed-spin chain with different next-nearest-neighbor AFM bonds (see figure 4a) [36].

\subsection{Thermodynamics}

Most of the experiments on quasi-1D mixed-spin systems carried out in the past concerned the magnetic susceptibility of ferrimagnetic chains with rather big $S_{1}$ spins (typically, $S_{1}=5 / 2$ ), making the system rather classical [37]. As a matter of fact, quantum effects are most pronounced in the extreme quantum case $\left(S_{1}, S_{2}\right)=(1,1 / 2)$, realized, for example, in the quasi-1D bimetallic material $\mathrm{NiCu}($ pba $\left.) \mathrm{D}_{2} \mathrm{O}\right)_{3} \cdot 2 \mathrm{D}_{2} \mathrm{O}[9]$. As mentioned above, this material has an exchange interaction of about $121 \mathrm{~K}$, and shows a three dimensional AFM ordering transition at $7 \mathrm{~K}$ (see figure 3) which somewhat obscures the very low temperature $(T)$ features of the $1 \mathrm{D}$ ferrimagnet. Still, lowfield measurements are in good agreement with the theoretically expected results. On the other hand, the magnetic fields necessary to reach the theoretically most interesting critical phase would be for this compound well beyond $100 \mathrm{~T}$, making the search for other compounds with weaker interactions an interesting challenge for experimentalists.

Low-temperature thermodynamics $\left(T \ll \varrho_{\mathrm{s}} M_{0}\right)$ of the mixed-spin chains is controlled by the FM magnons, so that in zero magnetic field it can be described by using Takahashi's constrained 


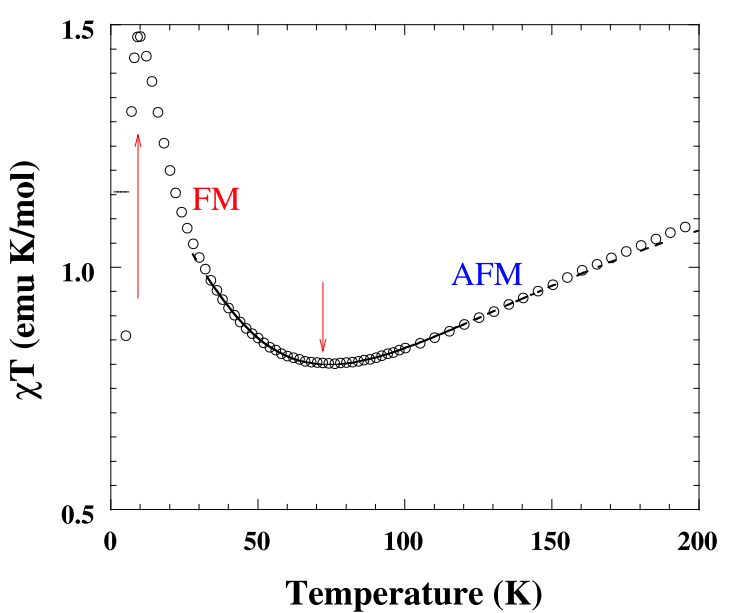

(a)

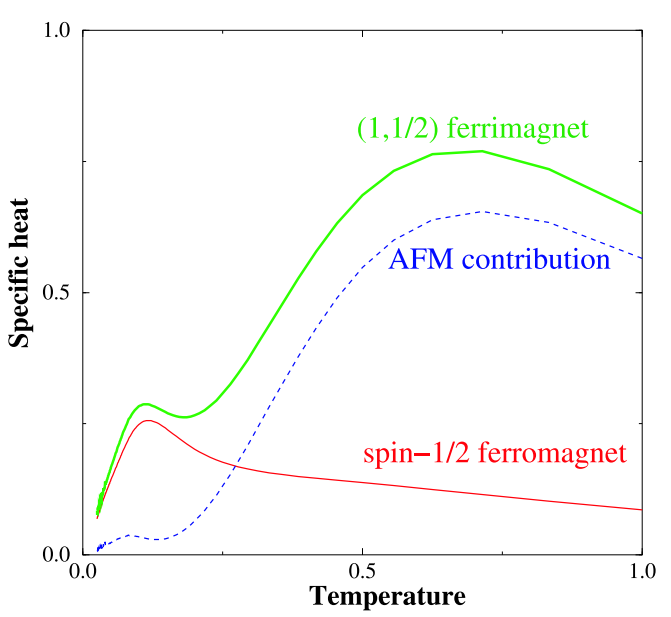

(b)

Figure 3. (a) Experimental results $(\chi \cdot T$ vs. $T)$ for the $(1,1 / 2)$ mixed-spin chain $\left.\mathrm{NiCu}(\mathrm{pba}) \mathrm{D}_{2} \mathrm{O}\right)_{3} \cdot 2 \mathrm{D}_{2} \mathrm{O}$ [9]. (b) $C_{v}$ vs. $T$ of the $(1 / 1 / 2)$ mixed-spin chain (DMRG) compared to $C_{v}(T)$ of the spin-1/2 FM chain (DMRG) at $h=0.05 J$. The difference of both specific heats can be identified as AFM contribution [32]. $C_{v}$ and $T$ are presented in the unites of $J$.

SWT for ferromagnets [40,41]. A few variants of this theory have also been applied to 1D ferrimagnets $[38,42,43]$. In terms of the ground-state parameters $\varrho_{\mathrm{s}}$ and $M_{0}$, the explicit form of the series in powers of $t \equiv T / \varrho_{\mathrm{s}} M_{0}$ for the uniform susceptibility $\chi$ and the specific heat $C_{v}$ read as [44]

$$
\begin{aligned}
& \frac{\chi T}{M_{0}}=\frac{2}{3} t^{-1}-\frac{\zeta\left(\frac{1}{2}\right)}{\sqrt{\pi}} t^{-\frac{1}{2}}+\frac{\zeta^{2}\left(\frac{1}{2}\right)}{2 \pi}+\mathcal{O}\left(t^{\frac{1}{2}}\right) \\
& \frac{C_{v}}{M_{0}}=\frac{3}{8} \frac{\zeta\left(\frac{3}{2}\right)}{\sqrt{\pi}} t^{\frac{1}{2}}-\frac{t}{2}+\left[\text { const }-\frac{15 \zeta\left(\frac{1}{2}\right)}{32 \sqrt{\pi}}\right] t^{\frac{3}{2}}+\mathcal{O}\left(t^{2}\right) .
\end{aligned}
$$

Here, $\zeta(z)$ is Riemann's zeta function, and const $=15\left(S_{1}^{2}+S_{1} S_{2}+S_{2}^{2}\right) \zeta\left(\frac{5}{2}\right) / 128 \sqrt{\pi}$.

As may be expected, the above expansions reproduce Takahashi's original expansions for the Heisenberg FM chain characterized by the parameters $M_{0}=S$ and $\varrho_{\mathrm{s}}=J S^{2}$. Apart from the coefficient of $t^{3 / 2}$ in the expansion for $C_{v}$, the above expressions reproduce precisely the thermodynamic Bethe-ansatz calculations for the spin-1/2 FM Heisenberg chain $[45,46]$. It is interesting to note that without the factor const in the expression for $C_{v}$, both expansions fulfill the general hypothesis according to which in 1D Heisenberg ferromagnets all observables should be universal functions of the bare couplings $M_{0}, \varrho_{\mathrm{s}}$, and $h$, realizing a no-scale-factor universality $[47,48]$. In particular, this means that the free-energy density should have the generic form

$$
\frac{F}{N}=T M_{0} \Phi_{F}\left(\frac{\rho_{s} M_{0}}{T}, \frac{h}{T}\right)
$$

where $\Phi_{F}(x, y)$ is a universal scaling function with no arbitrary scale factors: for example, the site spin $S$ enters only indirectly, through $\varrho_{\mathrm{s}}$ and $M_{0}$. The appearance of the factor violating the above scaling hypothesis is probably an artifact of Takahashi's SWT. As far as we know, the universal low- $T$ thermodynamic properties of quasi-1D quantum ferrimagnets have not been studied so far.

Whereas low- $T$ thermodynamics reflects the dispersion relation of the lower gapless FM branch $E_{k}^{-}$, the intermediate- $T$ behavior is dominated by that of the upper AFM branch $E_{k}^{+}$. Therefore, when the condition $T \ll \Delta^{+}$is violated, all the thermodynamic parameters are expected to demonstrate a crossover from a FM to a AFM behavior. In particular, for the uniform susceptibility $\chi$ one may expect $\chi(T) \propto T^{-2}$ (as $T \rightarrow 0$ ) and the Curie law behavior $\chi(T) \propto T^{-1}$ in the high- $T$ region. Figure 3a displays a typical experimental curve $(\chi T$ vs. $T)$ which is usually used to determine the character of the short-range interactions. For a paramagnetic system, Curie's law implies the 
behavior $\chi T=$ const over the whole $T$ range. If the magnetic system has dominant FM (AFM) interactions, $\chi T$ increases (decreases) when $T$ is decreased. Thus, in the interval $70 \mathrm{~K}<T<300 \mathrm{~K}$ the dominating coupling between nearest-neighbor spins is AFM, whereas the increase of $\chi T$ below the minimum around $T \approx 70 \mathrm{~K}$ implies that the system behaves like a FM chain at low $T$. The steep decrease below $10 \mathrm{~K}$ may be attributed to an established 3D AFM long-range order.

Turning to the behavior of the specific heat, it changes from $C_{v}(T) \propto T^{1 / 2}$ in the extreme low- $T$ region [see equation (3)], through an AFM Schottky-like peak (for intermediate $T$ ) to a paramagnetic $T^{-2}$ decay for high $T$ (see figure $3 \mathrm{~b}$ ). In addition, $C_{v}(T)$ acquires a characteristic double-peak structure for $0<h<h_{\mathrm{c} 1}$, which is related to the gapped modes $E_{k}^{ \pm}[38]$. As a matter of fact, both gapped antiferromagnets as well as ferromagnets in an external magnetic field exhibit a pronounced peak whose position is related to the gaps $\Delta^{ \pm}$. Such a double-peak structure has also been predicted for the $(1,1 / 2)$ chain with a FM exchange constant $(J<0)[39]$.

\section{Mixed-spin chains and ladders with magnetic frustration}

Over the last years there has been an increasing interest in quantum spin systems with competing exchange interactions [49]. Quasi-1D quantum spin systems with geometric frustration, both for half-integer and integer spins [50-54] (see also the review article [15]) set up an important part of this research. It is remarkable that up to now a relatively small amount of research on 1D frustrated mixed-spin models has been published. These models typically exhibit ground states with a net FM moment, so that a number of intriguing issues like the nature of the new phases as well as the character of the ferrimagnetic-paramagnetic transitions may be studied. What makes such transitions interesting is the fact that the order parameter is a conserved quantity: As is known, in systems with a Heisenberg $S O(3)$ symmetry this conservation law is expected to lead to strong constraints on the critical field theories $[55,56]$. Below we survey the available results for some generic Heisenberg spin models relevant to quasi-1D quantum ferrimagnets with competing interactions.

\subsection{Mixed-spin $J_{1}-J_{2}$ Heisenberg chains}

The mixed-spin Heisenberg chain composed of two types of alternating spins $\left(S_{1}>S_{2}\right)$, which are connected via competing nearest-neighbor $\left(J_{1}>0\right)$ and next-nearest-neighbor $\left(J_{2}, J_{2}^{\prime}>0\right)$ AFM exchange bonds (see figure 4a), is one of the simplest realistic models of frustrated 1D

(a)

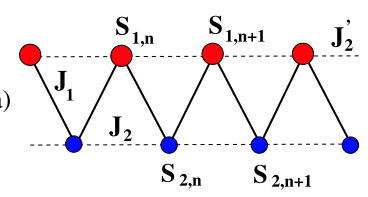

(b)

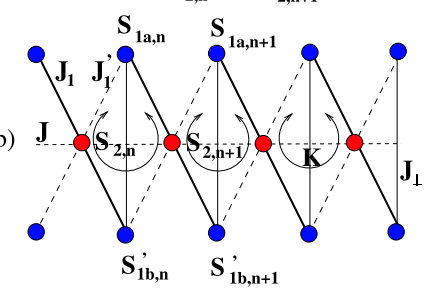

(c)

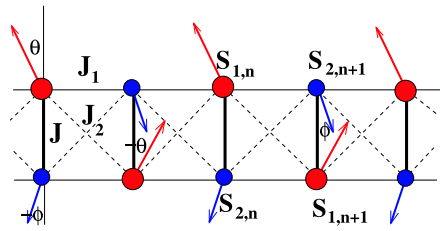

(d)

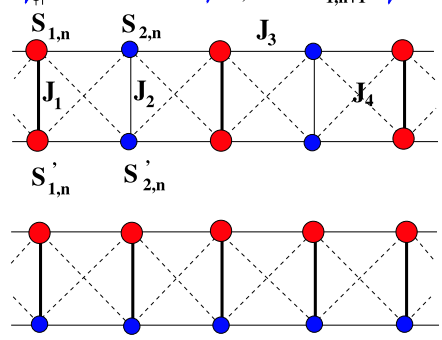

Figure 4. Some generic 1D Heisenberg spin models with AFM exchange bonds exhibiting ferrimagnetic ground states: (a) The mixed-spin $J_{1}-J_{2}$ chain with AFM nearest- $\left(J_{1}\right)$ and nextnearest-neighbor $\left(J_{2}, J_{2}^{\prime}\right)$ exchange bonds; (b) The distorted $\left(J_{1} \neq J_{1}^{\prime}\right)$ diamond chain with frustrated vertical bonds $\left(J^{\prime}\right)$ and four-spin cyclic exchange interactions $(K)$; (c,d,e) Three generic types of mixed-spin ladders with geometric frustration. 
quantum ferrimagnets $[22,36]$. This model may also be considered as a ferrimagnetic analogue of the frustrated FM Heisenberg chain with FM nearest-neighbor- $\left(J_{1}<0\right)$ and AFM next-nearestneighbor $\left(J_{2}>0\right)$ exchange bonds. The spin- $1 / 2$ frustrated $J_{1}-J_{2} \mathrm{FM}$ chain has recently attracted much attention, as it is supposed to describe a number of quasi-1D edge-sharing cuprates, such as $\mathrm{Rb}_{2} \mathrm{Cu}_{2} \mathrm{Mo}_{3} \mathrm{O}_{12}$ [57] and $\mathrm{LiCuVO}_{4}$ [58]. The latter material exhibits multiferroic properties [59] as well as an interesting specific phase transition in a magnetic field from an ordered spiral to an ordered modulated-collinear magnetic phase [60]. On the theoretical side, the latter FM model was shown to exhibit a vector chiral long-range oder (in a moderate magnetic field) as well as a rich variety of exotic quantum phases with different kinds of multipolar spin correlations (in a larger magnetic field) [61,62].

Turning to the mixed-spin $J_{1}-J_{2}$ chain, let us begin with a comment on its classical limit. The ground state of the model can be described by the ansatz $\mathbf{S}_{i, n}=S_{i}[\mathbf{u} \cos (Q n)+\mathbf{v} \sin (Q n)]$ $(i=1,2)$, where $\mathbf{u} \perp \mathbf{v}$ are unit vectors in the spin space. The classical ferrimagnetic state with a pitch angle between neighboring spins $Q / 2=\pi$ is stable up to the phase transition point $J_{2 c}=S_{1} S_{2} J_{1} /\left[2\left(S_{1}^{2}+S_{2}^{2}\right)\right]$. In the strongly frustrated region $J_{2}>J_{2 c}$, the stable state is a spiral with an ordering wave vector given by $\cos (Q / 2)=-S_{1} S_{2} J_{1} /\left[2 J_{2}\left(S_{1}^{2}+S_{2}^{2}\right)\right]$. In the limit $J_{2} \rightarrow \infty$, $Q=\pi$ and the system is composed of two decoupled AFM chains with site spins $S_{1}$ and $S_{2}$.

Next, let us discuss the extreme quantum case $\left(S_{1}, S_{2}\right)=(1,1 / 2)$. Already a qualitative semiclassical analysis implies that near the classical transition point at $J_{2}=0.2 J_{1}$ the FM magnon branch $E_{k}^{-}$(see figure 2) is strongly flattened, whereas the optical AFM branch shows only a smooth increase of the AFM gap $\Delta^{+}$. This means that the optical magnons do not play any important role in the mechanism of the transition. On approaching the classical transition point, the frustration and quantum spin fluctuations play somewhat different roles: Whereas the magnetic frustration strongly reduces the short-range spin correlation length in the ferrimagnetic phase, the quantum fluctuations stabilize the ferrimagnetic order up to the point $J_{2}=0.231 J_{1}$ beyond the classical transition point at $J_{2}=0.2 J_{1}$. Another effect of the quantum fluctuations is related to the change of the character of the classical transition: A detailed DMRG analysis of the low-energy levels around the classical transition clearly indicates a level crossing, i.e., a first-order quantum phase transition to a singlet ground state at $J_{2}=0.231 J_{1}$. Further, DMRG has also indicated that at least from $J_{2}=0.25 J_{1}$ upwards the discussed model exhibits a singlet ground state [22].

A number of open issues related to the mixed-spin $J_{1}-J_{2}$ Heisenberg model can be indicated. One of the important open problems concerns the nature of the singlet ground states established beyond the ferrimagnetic phase. Since in $1 \mathrm{D}$ systems the classically broken $\mathrm{SO}(3)$ symmetry in the spiral state is generally expected to be restored by quantum fluctuations, one is enforced to look for possible magnetically disordered states. A valuable information can be obtained from the Lieb-Schultz-Mattis theorem [63] adapted to mixed-chain spin models [64]. The theorem is applicable to systems with a half-integer cell spin $\left(S_{1}+S_{2}\right)$ and says that the model either has gapless excitations or else has degenerate ground states. Therefore, one may look for phases with presumably some broken discrete symmetry. In particular, the long-ranged chiral phase found in the frustrated spin-1/2 FM $J_{1}-J_{2}$ model in the small magnetic field region could be a possible candidate [61]. However, the variety of possible non-magnetic quantum phases may be considerably enlarged due to the existence of two kinds of site spins in the mixed variant of the parent model.

\subsection{Frustrated diamond chains}

The diamond Heisenberg chain is another generic model of 1D quantum ferrimagnets constructed from one kind of spins living on sublattices with different number of sites (see figure 4b). The frustrated symmetric diamond chain (SDC) $\left(J_{1}=J_{1}^{\prime}, J=0\right)$ with AFM vertical bonds $J_{\perp}>0$ is probably the first studied model of $1 \mathrm{D}$ quantum ferrimagnet with competing interactions $[65,66]$. A particular variant of the frustrated model, the distorted $\left(J_{1} \neq J_{1}^{\prime}\right)$ spin- $1 / 2$ diamond chain, has received an increasing theoretical $[67,68]$ as well as experimental interest in the past decade due to its rich quantum phase diagram [69] (see figure 5a) and the relevance to the real material $\mathrm{Cu}_{3}\left(\mathrm{CO}_{3}\right)_{2}(\mathrm{OH})_{2}$ (azurite) [70]. Without external magnetic field, three quantum phases in the parameter space $\left(J_{1} / J_{\perp}, J_{1}^{\prime} / J_{\perp}\right)$ have been discussed for the distorted model: For $J_{1} / J_{\perp}, J_{1}^{\prime} / J_{\perp} \ll 1$, 
the low-energy sector is governed by an effective spin-1/2 AFM Heisenberg model, which indicates the formation of a gapless spin-fluid phase (SF) with some additional high-energy modes related to local excitations of the vertical dimers. For intermediate $J_{1} / J_{\perp}$ and $J_{1}^{\prime} / J_{\perp}$, the ground state dimerizes, forming a twofold degenerate sequence of alternating tetramers and dimers $\left(\mathrm{TD}_{1}\right.$ phase). Finally, for both $J_{1} / J_{\perp}$ and $J_{1}^{\prime} / J_{\perp}$ sufficiently large, the ground state is a $1 \mathrm{D}$ ferrimagnet. These phases can be clearly identified already in the SDC: SF $\left(J_{1}<0.5 J_{\perp}\right)$, $\mathrm{TD}_{1}\left(0.5 J_{\perp}<J_{1}<1.10 J_{\perp}\right)$, and $1 \mathrm{D}$ ferrimagnet $\left(J_{1}>1.10 J_{\perp}\right)$.
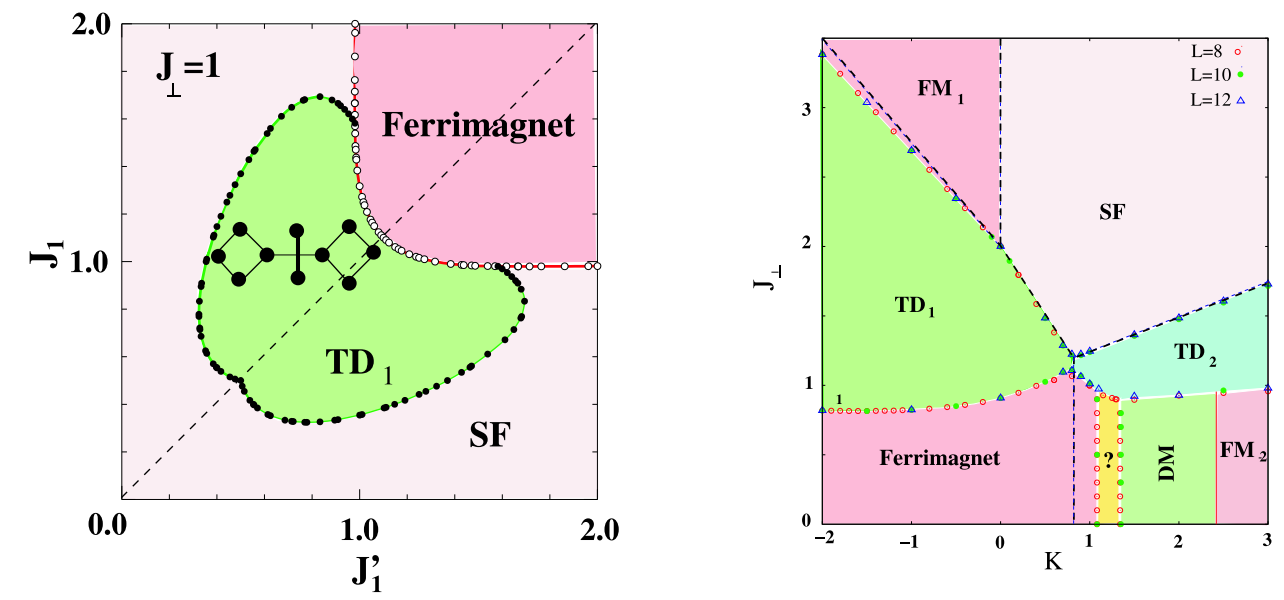

Figure 5. (a) Quantum phase diagram $(T=0)$ of the distorted diamond chain in the parameter space $J_{1}$ vs. $J_{1}^{\prime}\left(J_{\perp}=1\right)$ [69]. SF indicates the gapless spin-fluid phase, whereas $\mathrm{TD}_{1}$ marks the tetramer-dimer phase constructed from alternating dimers and tetramers in local singlet states. (b) Quantum phase diagram $(T=0)$ of a frustrated SDC with competing four-spin cyclic interactions in the parameter space $J_{\perp}$ vs. $K\left(J_{1}^{\prime}=J_{1}^{\prime}=1\right)$ [71]. In this system, there appear at least five new phases denoted as follows: $\mathrm{FM}_{1}$ (fully-polarized FM phase), $\mathrm{FM}_{2}$ (another phase with a net FM moment), DM (fourfold degenerate singlet phase), $\mathrm{TD}_{2}$ (another tetramer-dimer phase with tetramers in local triplet states), and an exotic non-Lieb-Mattis ferrimagnetic state denoted by a question mark. The dashed lines trace the phase boundaries of the single-diamond system.

The diamond Heisenberg chain is also one of the simplest quantum spin models admitting a four-spin cyclic exchange coupling. Below we discuss the impact of this competing interaction on the quantum phase diagram $(T=0)$ of the frustrated SDC [71]. The schematic Hamiltonian of the model is presented in figure $4 \mathrm{~b}$, the standard four-spin cyclic exchange coupling [72] in a single diamond being controlled by the parameter $K$. It is important to notice that the cyclic coupling does not violate the local symmetry of the frustrated $\left(J_{\perp}>0\right)$ SDC model under the exchange of pairs of off-chain spins $\mathbf{S}_{1 a, n}$ and $\mathbf{S}_{1 b, n}$ in the diamonds. Thus, in the important case of spin-1/2 offchain operators, the system is characterized (as in the frustrated model without cyclic interactions) by $L$ local good quantum numbers $s_{n}=0,1(n=1,2, \ldots, L)$ related to the composite off-chain spins $\mathbf{S}_{1, n}=\mathbf{S}_{1 a, n}+\mathbf{S}_{1 b, n}: \mathbf{S}_{1, n}^{2}=s_{n}\left(s_{n}+1\right)$. Using this local symmetry, the Hamiltonian of the $\mathrm{SDC}$ can be represented in the compact form

$$
\mathcal{H}_{c}=E_{0}+\sum_{n=1}^{L}\left[J_{1} \mathbf{S}_{1, n} \cdot\left(\mathbf{S}_{2, n}+\mathbf{S}_{2, n+1}\right)+J_{n} \mathbf{S}_{2, n} \cdot \mathbf{S}_{2, n+1}+\frac{K}{2}\left\{\mathbf{S}_{1, n} \cdot \mathbf{S}_{2, n}, \mathbf{S}_{1, n} \cdot \mathbf{S}_{2, n+1}\right\}\right] .
$$

Here $E_{0}=J_{\perp} \sum_{n=1}^{L}\left[s_{n}\left(s_{n}+1\right)-3 / 4\right]$ is a fixed number for every sector defined as a sequence of the local quantum numbers $\left[s_{1}, s_{2}, \ldots, s_{L}\right], J_{n}=J+K / 4-s_{n} K$, and $\{A, B\}$ is the anticommutator of the operators $A$ and $B$.

Let us briefly comment the phase diagram for the spin-1/2 SDC (figure 5b), by using some symmetries of the Hamiltonian (5). In the parameter space where the ground state is characterized by $s_{n}=0(n=1,2, \ldots, L)$, the first and third terms in the square brackets vanish and the model 
is equivalent to the spin- $1 / 2$ Heisenberg chain with an effective exchange parameter $J+K / 4$. This explains the presence of a fully polarized $\mathrm{FM}_{1}(J+K / 4<0)$ and a gapless spin-fluid $(J+K / 4>0)$ phases for large enough $J_{\perp} / J_{1}$. Note that besides the well-documented collective modes, these phases exhibit specific additional single-particle modes which are related to local triplet excitations on the vertical dimers. Being eigenstates of the Hamiltonian, these excitations are completely localized in the SDC.

For intermediate values of the parameter $J_{\perp} / J_{1}$, the ground state lies in the sector $[1,0, \cdots, 1,0]$. The tetramer-dimer phase $\mathrm{TD}_{1}$ studied in the DDC model (see figure $5 \mathrm{a}$ ) survives in the region with relatively small cyclic interactions. This doubly degenerate singlet state may roughly be thought of as a product of plaquette singlet states on every second diamond, as depicted in figure 5a. For larger $K$ at fixed $J_{\perp}$, there appears another tetramer-dimer phase $\left(\mathrm{TD}_{2}\right)$, with every second diamond approximately in a triplet state. The numerical ED analysis shows relatively strong AFM correlations between the neighboring triplet diamonds, as opposed to the $\mathrm{TD}_{1}$ state where the diamonds are weakly correlated. Clearly, both tetramer-dimer phases are gapped and doublydegenerated.

Finally, for $J_{\perp} / J_{1}<1$ the ground state lies in the sector $s_{n}=1(n=1,2, \ldots, L)$, so that the low-energy region of equation (5) describes a mixed-spin $(1,1 / 2)$ chain with competing three-spin exchange interactions. To the best of our knowledge, realistic 1D mixed-spin Heisenberg models with multiple-spin exchange interactions have not been discussed in the literature, although these interactions may play an important role in some recently synthesized mixed-spin magnetic materials and nanomagnets $[73,74]$. Here we restrict ourselves to a general overview of the spin phases of this interesting mixed-spin model with cyclic interactions in the extreme quantum case $\left(S_{1}, S_{2}\right)=$ $(1,1 / 2)$. We also suppose a FM effective exchange interactions between the in-chain spins (i.e., $\left.J_{n} \equiv J-3 K / 4<0\right)$. In the region $K>1.2 J_{1}$, a detailed numerical ED analysis indicates at least two additional phases, denoted by $\mathrm{DM}$ and $\mathrm{FM}_{3}$ in figure 5b [71]. DM is a dimerized singlet phase stabilized approximately in the region $1.5 J_{1} \leqslant K \leqslant 2.3 J_{1}$, whereas $\mathrm{FM}_{3}$ is a magnetic phase with a net FM moment. Finally, in the narrow interval $1.2 J_{1}<K<1.5 J_{1}$ a specific ferrimagnetic phase characterized by a reduced magnetic moment per cell, $M<M_{0}=S_{1}-S_{2}$, seems to appear. Below, other mixed-spin models exhibiting similar exotic (non-Lieb-Mattis) ferrimagnetic phases are surveyed.

\subsection{Mixed-spin ladders with geometric frustration}

Some typical examples of mixed-spin ladder structures are shown in figure 4 . The first two structures reproduce, e.g., arrangements of the magnetic atoms $\mathrm{Mn}\left(S_{1}=5 / 2\right)$ and $\mathrm{Cu}\left(S_{2}=1 / 2\right)$ along the a-axis in the compounds $\mathrm{MnCu}(\mathrm{pbaOH})\left(\mathrm{H}_{2} \mathrm{O}\right)_{3}$ (pbaOH $=2$-hydroxy-1,3- propylenebisoxamato) and $\mathrm{MnCu}(\mathrm{pba})\left(\mathrm{H}_{2} \mathrm{O}\right)_{3} \cdot 2 \mathrm{H}_{2} \mathrm{O}$ (pba $=1,3$-propylenebisoxamato), respectively. Along the caxis, the magnetic ions in both mixed-spin compounds are arranged as shown in figure 4(e) [2]. A very recently synthesized quasi-1D mixed-valent-iron material $\left[\mathrm{Fe}^{\mathrm{II}} \mathrm{Fe}^{\mathrm{III}}\right.$ (trans-1,4-cyclohexanedi-

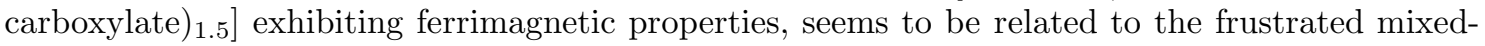
spin structure shown in figure 4d [75]. We are not aware of any real ferrimagnetic compound related to the ladder structure shown in figure 4e. Nevertheless, in view of the easy control of the molecularunit positions in the molecular chemistry, it may be expected that other materials, related to the generic mixed-spin models presented in figure 4, will be synthesized in the near future.

On the theoretical side, unfrustrated variants of the ladder models presented in figure 4 have already been analyzed in a number of publication [76-81]. The unfrustrated checkerboard ladder (figure $4 \mathrm{c}$ ) with AFM bonds $\left(J, J_{1}>0\right)$ exhibits a ferrimagnetic ground state. Its low-energy properties closely reproduce the properties of the generic mixed-spin chain discussed in section 2 [79]. Note that a variant of this structure with FM legs $\left(J_{1}<0\right)$ demonstrates completely different features: The FM leg coupling drives the system into a gapless spin-fluid ground state which is characteristic of the spin-1/2 AFM Heisenberg chain [81]. Similar gapless phase appears in the unfrustrated variant of the model displayed in figure $4 \mathrm{e}[77,78]$, whereas the model shown in figure $4 \mathrm{~d}$ possesses a gapped non-degenerate rung-singlet ground state [80], which is the characteristic phase of the uniform spin-1/2 Heisenberg ladder. 


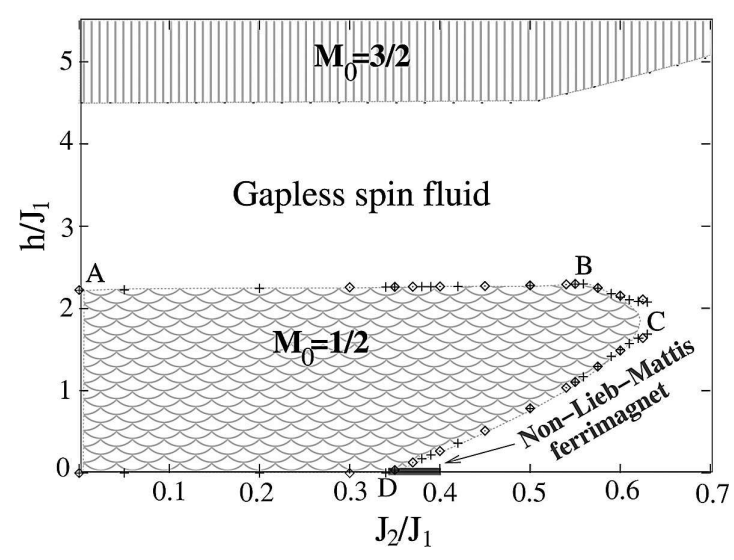

(a)

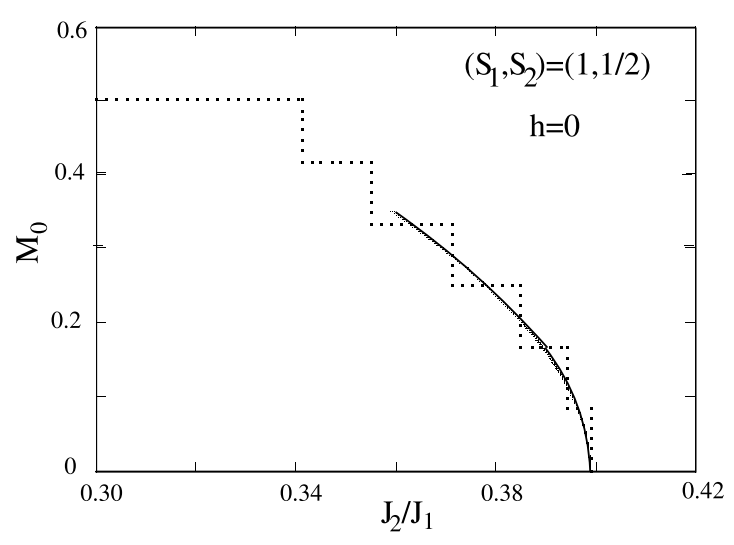

(b)

Figure 6. (a) Phase diagram of the checkerboard mixed-spin $(1 / 1 / 2)$ ladder (figure $4 c$ ) in the $\left(J_{2} / J_{1}, h / J_{1}\right)$ plane [82]. The phase boundary of the fully polarized phase $\left(M_{0}=3 / 2\right)$ is exact. Here $M_{0}$ is the FM moment per rung. The boundary (ABCD) of the ferrimagnetic $M_{0}=1 / 2$ plateau phase is obtained from numerical ED of periodic clusters with $L=10$ (squares) and $L=12$ (crosses) rungs. The points B, C, and D mark, respectively, the change of the lowest excited states, the tip of the lobe, and the $h=0$ transition point at $J_{2} / J_{1}=0.399$ from the $M_{0}<1 / 2$ ferrimagnetic phase to a gapless spin-fluid phases. The latter phase occupies the rest of the phase diagram. (b) $M_{0}$ vs. $J_{2} / J_{1}$ for the non-Lieb-Mattis (i.e., $M_{0}<1 / 2$ ) ferrimagnetic phase, as obtained from numerical ED of $L=12$ clusters (dashed line). The solid line connects the midpoints of the steps [83].

To understand the role of the geometric frustration in such mixed-spin models, let us turn to the phase diagram in figure 6 a presenting the phases of the frustrated $(1,1 / 2)$ checkerboard ladder (figure 4c) in the parameter space $\left(J_{2} / J_{1}, h / J_{1}\right)$ [82]. We are not aware of any publications studying the other two frustrated models displayed in figures $4 \mathrm{~d}$, and e. As a function of the frustration parameter $J_{2} / J_{1}$, the classical phase diagram of the frustrated checkerboard model exhibits three phases, which can be described by the angles $(\theta, \phi)$ fixing the directions of the classical spins $\mathbf{S}_{1, n}$ and $\mathbf{S}_{2, n}$ in respect to the classical ferrimagnetic configuration $(\theta, \phi)=(0,0)$ with up-S $\mathbf{S}_{1, n}$ and down- $\mathbf{S}_{2, n}$ spins. In the special case $\left(S_{1}, S_{2}\right)=(1,1 / 2)$, the classical canted state shown in figure $4 \mathrm{c}$ is stable in the interval $0.3219 J_{1}<J_{2}<0.4606 J_{1}$. For larger $J_{2}$, a collinear configuration with $(\theta, \phi)=(\pi / 2, \pi / 2)$ is stabilized. In the classical limit, the transitions between the canted state and the other two phases are continuous. Turning to the quantum model, the following changes in the quantum phase diagram (line $h=0$ in figure 6a) can be indicated. Whereas the classical ferrimagnetic phase survives quantum fluctuations, the collinear magnetic state is completely destroyed. Instead, for $J_{2}>0.399 J_{1}$ there appears a gapless spin-fluid phase. In the general chase, the quantum paramagnetic state is either critical (for half-integer $S_{1}+S_{2}$ ), or gapped (for integer $S_{1}+S_{2}$ ).

The most interesting changes appear in the classical canted state. In figure $6 \mathrm{~b}$ we show numerical ED results for the FM moment per rung $M_{0}$ as function of the frustration parameter $J_{2} / J_{1}$. We see that the quantum phase which substitutes the classical canted phase is characterized by a finite FM moment which is, however, less than the quantized FM moment per rung $\left(S_{1}-S_{2}=1 / 2\right)$ of a standard Lieb-Mattis ferrimagnet. Notice that this phase exists only at $h=0$. For $h>0$ it merges into the Luttinger liquid phase (see figure 6a). An extrapolation of the ED results for $L=8,10$ and 12 rungs definitely indicates the presence of this phase in the interval $0.341 J_{1}<$ $J_{2}<0.399 J_{1}$. Already a qualitative semiclassical analysis (supported by ED results) implies that on approaching the phase transition point at $J_{2}=0.341 J_{1}$ from the ferrimagnetic phase, the lower magnon branch $E_{k}^{-}$softens in the vicinity of $k=\pi$, and the gap at $k=\pi$ vanishes at the transition point to the canted phase. Thus, there appears a linear Goldstone mode which is 
characteristic of the classical canted phase. It may be suggested that this critical mode survives quantum fluctuations [55], whereas the spin rotation symmetry $U(1)$ in the $x y$ plane should be restored, i.e., $\left\langle S_{1, n}^{x}\right\rangle=\left\langle S_{2, n}^{x}\right\rangle=0$. This scenario with a power-law decay of the transverse spin-spin correlations is supported by the renormalization-group analysis of similar phases in quantum rotor models [55] as well as by a recent DMRG analysis of a similar phase found in a generalized SDC model with an additional competing AFM interaction between the off-chain spins in figure 4b [84]. The first studied quantum spin model exhibiting such an exotic quantum state seems to be the spin-1/2 two-leg ladder constructed from different (one FM and another AFM) legs and AFM rungs [85]. Quite recently, there has been a number of reports indicating similar 1D quantum magnetic phases in some decorated quantum spin chains [86,87] as well as in a mixed-spin $(2,1)$ Heisenberg chain with competing single-ion anisotropies [88].

\section{Conclusion}

In conclusion, we have surveyed the available theoretical results related to some generic quantum spin models displaying 1D ferrimagnetic ground states. The stress was put on the interplay between quantum fluctuations and the competing interactions. The discussed models of quasi-1D ferrimagnets with competing interactions exhibit a rich variety of magnetic and paramagnetic quantum phases and provide unique examples of $1 \mathrm{D}$ magnetic-paramagnetic quantum phase transitions. Finally, a great deal of important open issues deserving further studies have been debated.

\section{Acknowledgements}

The author thanks the staff of the Fakultät für Physik, Universität Bielefeld for hospitality and Jürgen Schnack for the critical reading of the manuscript. This work was supported by Deutsche Forschungsgemeinschaft (Grant SCHN 615/13-1) and Bulgarian Science Foundation (Grant D002$264 / 18.12 .08)$.

\section{References}

1. Wolf W.P., Rep. Prog. Phys., 1961, 24, 212.

2. Kahn O. Molecular Magnetism. VCH, New York, 1993.

3. Verdaguer M., Gleizes A., Renard J.P., Seiden J., Phys. Rev. B, 1984, 29, 5144.

4. Gleizes A., Verdaguer M., J. Am. Chem. Soc., 1984, 106, 3727.

5. Pei Y., Verdaguer M., Kahn O., Sletten J., Renard J.P., Inorg. Chem., 1987, 26, 138.

6. Kahn O., Pei Y., Verdaguer M., Renard J.-P., Sletten J., J. Am. Chem. Soc., 1988, 110, 782.

7. Koningsbruggen P.J., Kahn O., Nakatani K., Pei Y., Renard J.-P., Legoll P., Inorg. Chem., 1990, 29, 3325.

8. Kahn O, Bakalbassis E., Mathoniere C., Hagiwara M., Katsumata K., Ouahab L., Inorg. Chem., 1997, 36, 1530.

9. Hagiwara M., K. Minami K., Narumi Y., Tatani K., Kindo K., J. Phys. Soc. Jpn., 1998, 67, 2209.

10. Clérac R., Miyasaka H., Yamashita M., Coulon C., J. Am. Chem. Soc., 2002, 124, 12837.

11. Effenberger H., J. Solis State Chem., 1999, 142, 6.

12. Ohta H., Okubo S., Kamikawa T., Kunimoto T., Inagaki Y., Kikuchi H., Saito T, Azuma M., Takano M., J. Phys. Soc. Jpn., 2003, 72, 2464.

13. Kikuchi H., Fujii Y., Chiba M., Mitsudo S., Idehara T., Tonegawa T., Okamoto K., Sakai T., Kuwai T., Ohta H., Phys. Rev. Lett., 2005, 94, 227201.

14. Nishide H., Adv. Mater., 1995, 7, 937.

15. Mikeska H.-J., Kolezhuk A.K. One-dimensional magnetism. - In: Quantum Magnetism (eds. Schollwöck U., Richter J., Farnell D.J.J., and Bishop R.F.). Springer-Verlag, Berlin Heidelberg, p. 1-83, 2004.

16. Lieb E.H., Mattis D., J. Math. Phys., 1962, 3, 749.

17. Ivanov N.B., Sen D. Spin wave analysis of Heisenberg magnets in restricted geometries. - In: Qantum Magnetism (eds. Schollwöck U, Richter J., Farnell D.J.J., and Bishop R.F.). Springer-Verlag, Berlin Heidelberg, p. 195-226, 2004. 
18. Pati S.K., Ramasesha S., Sen D., Phys. Rev. B, 1997, 55, 8894.

19. Brehmer S., Mikeska J.-H., Yamamoto S., J. Phys.: Condens. Matter, 1997, 9, 3921.

20. Pati S.K., Ramasesha S., Sen D., J. Phys.: Condens. Matter, 1997, 9, 8707.

21. Yamamoto S., Brehmer S., Mikeska H.-J, Phys. Rev. B, 1998, 57, 13610.

22. Ivanov N.B., Richter J., Schollwöck U., Phys. Rev. B, 1998, 58, 14456.

23. Ivanov N.B., Phys. Rev. B, 1998, 57, R14024.

24. Kolezhuk A.K., Mikeska H.-J, Yamamoto S., Phys. Rev B, 1997, 55, R3336.

25. Kolezhuk A.K., Mikeska H.-J, Maisinger K., Schollwöck U., Phys. Rev. B, 1999, 59, 13565.

26. Halperin B.I., Hohenberg P.C., Phys. Rev., 1969, 188, 898.

27. Ivanov N.B., Phys. Rev. B, 2000, 62, 3271.

28. Zheng W., Oitmaa J., Phys. Rev. B, 2003, 67, 224421.

29. Affleck I., Phys. Rev. B, 1990, 41, 6697.

30. Giamarchi T., Tsvelik A.M., Phys. Rev. B, 1999, 59, 11398.

31. Giamarchi T., Rüegg Ch., Tchernyshov O., Nature Physics, 2008, 4, 198.

32. Maisinger K., Schollwöck U., Brehmer S., Mikeska J.-H., Yamamoto S., Phys. Rev. B, 1998, 58, R5908.

33. Alcaraz F.C., Malvezzi A.L., J. Phys. A: Math. Gen., 1997, 30, 767.

34. Sakai T., Yamamoto S., Phys. Rev. B, 1999, 60, 4053.

35. Sakai T., Okamoto K., Phys. Rev. B, 2002, 65, 214403.

36. Kuramoto T., J. Phys. Soc. Jpn., 2004, 73, 2518.

37. Kahn O., Pei Y., Journaux Y. Inorganic Materials. Wiley, New York, 1995.

38. Yamamoto S., Fukui T., Maisinger K., Schollwöck U., J. Phys.: Condens. Matter, 1998, 10, 11033.

39. Fukushima N., Honecker A., Wessel S., Brenig W., Phys. Rev. B, 2004, 69, 174430.

40. Takahashi M., Prog. Theor. Phys. Supp., 1986, 87, 233.

41. Takahashi M., Phys. Rev. Lett., 1987, 58, 168.

42. Yamamoto S., Fukui T., Phys. Rev. B, 1998, 57, R14008.

43. Wu C., Chen B., Dai X., Yu Y., Su Z.B., Phys. Rev. B, 1999, 60, 1057.

44. Ivanov N.B., Schollwöck U. Quantum Heisenberg ferrimagnetic chains - In: Contemporary Problems in Condensed Matter Physics. Nova Science Publishers Inc., New York, 2001.

45. Takahashi M., Yamada M., J. Phys. Soc. Jpn., 1985, 54, 2808.

46. Yamada M., Takahashi M., J. Phys. Soc. Jpn., 1986, 55, 2024.

47. Read N., Sachdev S., Phys. Rev. Lett., 1995, 75, 3509.

48. Takahashi M., Nakamura H., Sachdev S., Phys. Rev. B, 1996, 54, R744.

49. Proc. Int. Conference on Highly frustrated magnetism, 7-12 September 2008, Braunschweig, Germany (J. Phys.: Conference Series, 145, 2009).

50. Bursill R., Gehring G.A., Farnell D.J.J., Parkinson J.B., Xiang T., Zeng C., J. Phys.: Condens. Matter, 1995, 7, 8605 .

51. Chitra R., Pati S., Krishnamurthy H.R., Sen D., Ramasesha S., Phys. Rev. B, 1995, 52, 6581.

52. Schollwöck U., Jolicoeur Th., Garel T., Phys. Rev. B, 1996, 53, 3304.

53. Kolezhuk A., Roth R., Schollwöck U., Phys. Rev. Lett., 1996, 77, 5142.

54. White S.R., Affleck I., Phys. Rev. B, 1996, 54, 9862.

55. Sachdev S., Senthil T., Ann. Phys. (N.Y.), 1996, 251, 76.

56. Sachdev S., Z. Phys. B, 1994, 94, 469.

57. Hase M., Kuroe H., Ozawa K., Suzuki O., Kitazawa H., Kido G., Sekine T., Phys. Rev. B, 2004, 70, 104426.

58. Enderle M. et al., Europhys. Lett., 2005, 70, 237.

59. Naito Y. et al., J. Phys. Soc. Jpn., 2007, 76, 023708.

60. Banks M.G. et al., J. Phys.: Condens. Matter, 2007, 19, 145227.

61. Hikihara T., Kecke L., Momoi T., Furusaki A., Phys. Rev. B, 2008, 78, 144404.

62. Sudan J., Lüscher A., Läuchli A. Preprint arXiv:0807.1923v1, 2008 (unpublished).

63. Lieb E.H., Schultz T., Mattis D.J., Ann. Phys. (N.Y.), 1961, 16, 407.

64. Fukui T., Kawakami N., Phys. Rev. B, 1997, 55, R14709.

65. Takano K., Kubo K., Sakamoto H., J. Phys.: Condens. Matter, 1996, 8, 6405.

66. Niggemann H., Uimin G., Zittartz J., J. Phys.: Condens. Matter, 1997, 9, 9031.

67. Okamoto K., Tonegawa T., Takahashi Y., Kaburagi M., J. Phys.: Condens. Matter, 1999, 11, 10485; Okamoto K., Tonegawa T., Kaburagi M., ibid., 2003, 15, 5979.

68. Mikeska H.-J., Luckmann C., Phys. Rev. B, 2008, 77, 054405.

69. Tonegawa T., Okamoto K., Hikihara T., Takahashi Y., Kaburagi M., J. Phys. Soc. Jpn., 2000, 69, 332.

70. Rule K.C. et al., Phys. Rev. Lett., 2008, 100, 117202. 
71. Ivanov N.B., Richter J., Schulenburg J., Phys. Rev. B, 2009, 79, 104412.

72. See, for example, Müller M., Vekua T., Mikeska H.-J., Phys. Rev. B, 2002, 66, 134423.

73. Kostyuchenko V.V. et al., Phys. Rev. B, 2003, 67, 184412.

74. Schnack J. Molecular magnetism. - In: Qantum Magnetism (eds. Schollwöck U., Richter J., Farnell D.J.J., and Bishop R.F.). Springer-Verlag, Berlin Heidelberg, p. 155-194, 2004

75. Zheng Yan-Zhen et al., Inorg. Chem., 2009, 48, 2028.

76. Sénéchal D., Phys. Rev. B, 1997, 52, 15319.

77. Fukui T., Kawakami N., Phys. Rev. B, 1998, 57, 398; Koga A., Kumada S., Fukui T., J. Phys. Soc. Jpn., 1998, 67, 622.

78. Langari A., Abolfath M., Martin-Delgado M.A., Phys. Rev. B, 2000, 61, 343; Langari A., MartinDelgado M.A., Phys. Rev. B, 2000, 62, 11725.

79. Ivanov N.B., Richter J., Phys. Rev. B, 2001, 63, 144429.

80. Trumper A.E., Gazza C., Phys. Rev. B, 2001, 64, 134408; Physica B, 2002, 320, 340.

81. Aristov D.N., Kiselev M.N., Phys. Rev. B, 2004, 70, 224402.

82. Ivanov N.B., Richter J., Phys. Rev. B, 2006, 73, 132407.

83. Ivanov N.B., Richter J., Phys. Rev. B, 2004, 63, 144429.

84. Montenegro-Filho R.R., Coutinho-Filho M.D., Phys. Rev. B, 2008, 78, 014418.

85. Tsukano M., Takahashi M., J. Phys. Soc. Jpn., 1997, 66, 1153.

86. Yoshikawa S., Miyashita S., J. Phys. Soc. Jpn., 2005, 74, 71.

87. Hida K., J. Phys. Soc. Jpn., 2007, 76, 024714; J. Phys.: Condens. Matter, 2007, 19, 145225; Hida K., Takano K., Phys. Rev. B, 2008, 78, 064407.

88. Tonegawa T., Okamoto K., Sakai T., Kaburagi M., J. Phys.: Conference Series, 2009, 145, 012066.

\title{
Спінові моделі квазіодновимірних квантових ферімагнетиків з конкуруючими взаємодіями
}

\author{
Н.Б. Іванов ${ }^{1,2}$ \\ 1 Інститут фізики твердого тіла, Болгарська академія наук, \\ 72 Царіградско шосе, 1784 Софія, Болгарія \\ 2 Факультет фізики, Університет Білєфельда, D-33501 Білєфельд, Німеччина \\ Отримано 19 червня 2009 р.
}

Ми представляємо короткий огляд недавньої теоретичної роботи, пов'язаної з загальними спіновими моделями Гайзенберга, що описують квазіодновимірні квантові ферімагнетики. Наголос зроблено на квантових ланцюжках і драбинках з сильною конкуренцією взаємодій, таких, як фрустрований $J_{1}-J_{2}$ ланцюжок зі змінними $(1,1 / 2)$ спінами, спін-1/2 ромбічний ланцюжок з чотириспіновими циклічними взаємодіями і деякі загальні типи драбинок з геометричними фрустраціями з різними значеннями спіна. Як правило, обговорювані моделі виявляють багаті квантові фазові діаграми і становлять цікаві приклади одновимірних квантових фазових переходів магнетик - парамагнетик. Оговорюються деякі відкритті проблеми у цій області досліджень.

Ключові слова: квантові спінові ланцюжки, ферімагнетики, фрустрації

PACS: $75.10 . J \mathrm{~m}, 75.10 \mathrm{Pq}, 75.45 .+j$ 
